\title{
Generalized nematohydrodynamic boundary conditions with application to bistable twisted nematic liquid-crystal displays
}

\author{
Angbo Fang, ${ }^{1}$ Tiezheng Qian, ${ }^{2}$ and Ping Sheng ${ }^{1}$ \\ ${ }^{1}$ Department of Physics and the Institute of Nano Science and Technology, Hong Kong University of Science and Technology, \\ Clear Water Bay, Kowloon, Hong Kong, China \\ ${ }^{2}$ Department of Mathematics, Hong Kong University of Science and Technology, Clear Water Bay, Kowloon, Hong Kong, China
}

(Received 24 September 2008; published 8 December 2008)

\begin{abstract}
Parallel to the highly successful Ericksen-Leslie hydrodynamic theory for the bulk behavior of nematic liquid crystals (NLCs), we derive a set of coupled hydrodynamic boundary conditions to describe the NLC dynamics near NLC-solid interfaces. In our boundary conditions, translational flux (flow slippage) and rotational flux (surface director relaxation) are coupled according to the Onsager variational principle of least energy dissipation. The application of our boundary conditions to the truly bistable $\pi$-twist NLC cell reveals a complete picture of the dynamic switching processes. It is found that the thus far overlooked translationrotation dissipative coupling at solid surfaces can accelerate surface director relaxation and enhance the flow rate. This can be utilized to improve the performance of electro-optical nematic devices by lowering the required switching voltages and reducing the switching times.
\end{abstract}

DOI: 10.1103/PhysRevE.78.061703

PACS number(s): 42.70.Df, 47.57.Lj, 47.50.-d

\section{INTRODUCTION}

The widely used nematic liquid crystal (NLC) displays consist of a liquid-crystal layer confined between two substrate surfaces. Currently, most nematic displays rely mainly on voltage-induced reorientation of the average molecular direction (director) within the bulk of the NLC layer. With the downsizing of NLC cells and the possibility of engineering the textures of substrates, surface effects become significant and may lead to improved performance of NLC cells. The bulk behavior of NLC layers is well described by the celebrated Ericksen-Leslie hydrodynamic theory [1]. However, parallel to the usually assumed no-slip boundary conditions for simple fluids, the so-called strong (rigid) anchoring conditions are often imposed in the study of NLC orientational dynamics; these are unable to treat the dynamic effects due to certain fluid-solid interactions.

Only a few works have been dedicated to describe the weak anchoring dynamics [2-4], in which a phenomenological surface viscosity $l_{r} \gamma_{1}$ is introduced, with $l_{r}$ a characteristic length and $\gamma_{1}$ the rotational viscosity in the bulk. A dynamic equation for director relaxation at a surface was proposed in the form of $l_{r} \gamma_{1} \mathbf{n}_{s} \times \dot{\mathbf{n}}_{s}=\mathbf{K}_{s}+\mathbf{K}_{a}$, where $\dot{\mathbf{n}}_{s}$ is the relaxation rate of the surface director and $\mathbf{K}_{s}$ is the torque produced by the substrate, obtainable from a modeling potential for anchoring energy such as the one in RapiniPapoular form. $\mathbf{K}_{a}$ is from the bulk fluid and is composed of the elastic torque and the viscous torque whose forms are specified according to the Frank-Oseen and Ericksen-Leslie theories [1], respectively.

This paper is organized as follows. In Sec. II we first derive the general boundary conditions for NLC flows according to the Onsager variational principle of least energy dissipation [5], to take care of various surface effects such as weak anchoring, slippage, rotational friction, and their interplays. Then in Sec. III we apply the general boundary conditions to study the truly bistable twisted nematic cells, where surface dynamics dominates the switching behavior between the topologically nonequivalent zero-twist state and $\pi$-twist state [6-10]. In the last section we summarize our main results and give relevant conclusions.

\section{GENERALIZED NEMATOHYDRODYNAMIC BOUNDARY CONDITIONS}

We recall that the core expressions for the Ericksen-Leslie hydrodynamic theory are the two constitutive equations for viscous stress and frictional molecular field (or torque), which in turn are obtained from the linear relations between dissipative forces and fluxes compatible with the local symmetry of the NLC:

$$
\begin{gathered}
\sigma_{\alpha \beta}^{v}=\alpha_{1} n_{\alpha} n_{\beta} n_{\mu} n_{\rho} A_{\mu \rho}+\alpha_{4} A_{\alpha \beta}+\alpha_{5} n_{\alpha} n_{\mu} A_{\mu \beta} \\
+\alpha_{6} n_{\beta} n_{\mu} A_{\mu \alpha}+\alpha_{2} n_{\alpha} N_{\beta}+\alpha_{3} n_{\beta} N_{\alpha}, \\
h_{\mu}=\gamma_{1} N_{\mu}+\gamma_{2} n_{\alpha} A_{\alpha \mu},
\end{gathered}
$$

where $\sigma_{\alpha \beta}^{v}$ is the total viscous stress, $h_{\mu}$ the frictional molecular field (with $\mathbf{n} \times \mathbf{h}$ giving the corresponding torque), $A_{\alpha \beta}=\left(\partial_{\alpha} v_{\beta}+\partial_{\beta} v_{\alpha}\right) / 2$ the symmetrized strain rate, and $\mathbf{N}=\dot{\mathbf{n}}$ $-\frac{1}{2}(\boldsymbol{\nabla} \times \mathbf{v}) \times \mathbf{n}$ the rate of change of the director with respect to the background flow. These two equations not only provide the expressions for the various forces (e.g., those in $\mathbf{K}_{a}$ ) acting on the boundary layer from the bulk NLC, but also suggest the appropriate forms of the frictional forces due to the solid substrates.

The rate of energy dissipation per unit volume in the bulk can be written as

$$
T \dot{S}=\sigma_{\alpha \beta}^{v} \partial_{\alpha} v_{\beta}+h_{\mu} \dot{n}_{\mu} .
$$

Substituting Eqs. (1) and (2) into (3), we can identify three types of terms involving $\dot{\mathbf{n}}$ :

$$
\gamma_{1} \dot{\mathbf{n}}^{2}, \quad 2 \alpha_{2} n_{\alpha} \dot{n}_{\beta} \partial_{\alpha} v_{\beta}, \quad 2 \alpha_{3} n_{\beta} \dot{n}_{\alpha} \partial_{\alpha} v_{\beta},
$$

where the Parodi relation for viscosity coefficients has been used to recombine similar terms. We note that the first term 
describes pure rotational friction, while the second and third terms reflect the cross coupling between the two different fluxes (the velocity gradient and the varying rate of director). Since $\alpha_{3}$ is typically much smaller than $\alpha_{2}$, it is reasonable to neglect the third term in most cases. The other four types of terms involve only the velocity-gradient flux and among them the canonical shear viscous term involving $\alpha_{4}$ is the most important and identical to the bulk dissipation for simple fluid, with $\alpha_{4} / 2$ corresponding to the usual shear viscosity.

In Ref. [11] a variational approach based on the Onsager principle is used to derive the boundary conditions for the moving contact line hydrodynamics, which proves to be capable of quantitatively resolving the long-standing issue about the incompatibility between the moving contact line and the no-slip boundary condition [12]. For the singlecomponent simple fluid, the rate of viscous dissipation in the bulk is $R_{v}=\int d V(\eta / 2)\left(\partial_{i} v_{j}+\partial_{j} v_{i}\right)^{2}$. Consider a boundary layer of fluid at the solid surface. The layer thickness is $\delta$ along the $z$ direction (normal to the fluid-solid interface). $R_{v}$ can be reduced to a surface integral $\int d S\left[(\eta / \delta)\left(\Delta v_{\tau}\right)^{2}\right]$, where $\Delta v_{\tau}$ $=\delta \partial_{z} v_{\tau}$ is the variation of the tangential velocity $v_{\tau}$ across the boundary layer. This immediately suggests that the surface dissipation should have the form

$$
R_{s}=\int d S \beta\left(v_{\tau}^{\text {slip }}\right)^{2},
$$

where the slip velocity $v_{\tau}^{\text {slip }}$ measures the tangential velocity difference between the fluid and the solid substrate. The slip coefficient $\beta$ is related to the bulk viscosity by $\beta=\eta / l_{s}$, with $l_{s}$ being the so-called slip length. Compared to the surface layer thickness $\delta$ mainly determined by the short-range fluidsolid repulsive interaction, the slip length $l_{s}$ mainly depends on the fluid-solid attractive interaction of longer range. For the continuum description to be valid, the surface layer thickness must be the smallest length scale [11]. In the noslip limit $l_{s}$ goes to zero, while $l_{s}=\infty$ corresponds to a free surface where the slip coefficient $\beta$ is zero. By minimizing the total dissipation functional $R_{v}+R_{s}$ with respect to the variation of the velocity field, we can obtain at the same time the Stokes equation and the Navier boundary condition [11]. The latter is of the form

$$
\eta\left(\partial_{m} v_{\tau}+\partial_{\tau} v_{m}\right)+\beta v_{\tau}^{\text {slip }}=0
$$

where $m$ and $\tau$ refer to the component indices normal (outward) and tangential to the surface, respectively.

Now we want to obtain the boundary conditions at the NLC-solid interface. The crucial step is to identify the appropriate forms of the dissipation functional at the NLCsolid interface for the application of the Onsager variational principle of least energy dissipation [5]. By performing analysis similar to the simple fluid, the $\alpha_{4}$ term in the bulk dissipation should be mapped to the slip-velocity term. The $\gamma_{1}$ term is a quadratic function of the flux $\dot{\mathbf{n}}$ describing the pure rotational friction. Mapped to the surface, its form should remain the same, but its coefficient should be modified by multiplying the bulk rotational viscosity with a length scale $l_{r}$ reflecting the range of the NLC-solid dissipative cou- pling. The cross term describing the coupling between the two fluxes, $\mathbf{v}^{\text {slip }}$ and $\dot{\mathbf{n}}$, can be obtained from the bulk dissipation term involving $\alpha_{2}$, by mapping $\partial_{z} v_{\tau}$ to $-\delta^{-1}(\hat{\mathbf{m}} \cdot \hat{\mathbf{z}}) v_{\tau}^{\text {slip }}$, where $\hat{\mathbf{m}} \cdot \hat{\mathbf{z}}$ equals +1 for the upper surface and -1 for the lower surface. In summary, the surface dissipation functional (which by definition is half of the rate of energy dissipation) should have the quadratic form

$$
R\left[\dot{n}_{\alpha}, v_{\tau}\right]=\frac{1}{2} \gamma_{1} l_{r}(\dot{\mathbf{n}})^{2}+\frac{1}{2} \beta\left(\mathbf{v}^{\mathrm{slip}}\right)^{2}+\gamma(\hat{\mathbf{m}} \cdot \mathbf{n})\left(\mathbf{v}^{\mathrm{slip}} \cdot \dot{\mathbf{n}}\right),
$$

where $\gamma$ is introduced to describe the coupling of the translational and rotational fluxes at the surface. It is of the dimension of bulk viscosity and of positive value since the $\alpha_{2}$ coefficient is negative and an extra negative sign is introduced in the mapping from the bulk dissipation term involving $\alpha_{2}$. We comment that for the surface dissipation functional to be positive definite, the following inequality must be satisfied:

$$
\gamma<\sqrt{\frac{l_{r}}{l_{s}}} \sqrt{\gamma_{1} \alpha_{4} / 2}
$$

where we have replaced $\beta$ by $\alpha_{4} / 2 l_{s}$. This inequality has been numerically verified by varying the ratio of $l_{r}$ and $l_{s}$. Violation of it (a too large $\gamma$ ) fails to produce steady flows.

From the surface dissipation functional we obtain the surface frictional force (which is tangential)

$$
F_{\tau}^{\mathrm{sf}}=-\beta v_{\tau}^{\mathrm{slip}}-\gamma(\hat{\mathbf{m}} \cdot \mathbf{n}) \dot{n}_{\tau}
$$

and the surface frictional molecular field

$$
h_{\alpha}^{\mathrm{sf}}=-\gamma_{1} l_{r} \dot{n}_{\alpha}-\gamma(\hat{\mathbf{m}} \cdot \mathbf{n}) v_{\alpha}^{\mathrm{slip}} .
$$

The tangential viscous stress acting on the boundary layer from the bulk NLC is just $-\sigma_{m}^{v}$. On the other hand, there is no corresponding frictional torque (or molecular field) exerted by the bulk NLC due to the fact that the bulk dissipation functional does not involve the spatial derivatives of $\dot{\mathbf{n}}$.

Now we are ready to obtain the complete boundary conditions for a nematic boundary layer by adding elastic contributions to the surface forces and torques. From the FrankOseen free-energy density

$$
\begin{aligned}
f_{d}= & \frac{1}{2}\left\{K_{1}(\boldsymbol{\nabla} \cdot \mathbf{n})^{2}+K_{2}[\mathbf{n} \cdot(\boldsymbol{\nabla} \times \mathbf{n})+2 \pi / p]^{2}\right. \\
& \left.+K_{3}[\mathbf{n} \times(\boldsymbol{\nabla} \times \mathbf{n})]^{2}\right\},
\end{aligned}
$$

the elastic stress is given by

$$
\sigma_{\alpha \beta}^{d}=-\pi_{\alpha \gamma}^{d} \partial_{\beta} n_{\gamma}
$$

with $\pi_{\alpha \beta}^{d}=\partial f_{d} / \partial\left(\partial_{\alpha} n_{\beta}\right)$. The elastic molecular field is

$$
h_{\alpha}^{d}=-\frac{\partial f_{d}}{\partial n_{\alpha}}+\partial_{\beta} \pi_{\beta \alpha}^{d} .
$$

We have another source of conservative force, i.e., the force due to the anchoring potential, $W[\mathbf{r}, \mathbf{n}]$, which contributes to the tangential force by $\partial_{\tau} W$ (a Marangoni-type force per unit area) and to the molecular field by $-\partial W / \partial n_{\alpha}$. Including all 
the tangential forces and requiring them to be balanced, we have the first general boundary condition at the NLC-solid interface:

$$
-\sigma_{m \tau}^{d}+\partial_{\tau} W-\sigma_{m \tau}^{v}-\beta v_{\tau}^{\text {slip }}-\gamma(\hat{\mathbf{m}} \cdot \mathbf{n}) \dot{n}_{\tau}=0,
$$

which may be called the generalized slip boundary condition. Including all the molecular fields and requiring them to be balanced, we obtain the second general boundary condition which may be called the rotational boundary condition:

$$
-\pi_{m \alpha}^{d}-\frac{\partial W}{\partial n_{\alpha}}-\gamma_{1} l_{r} \dot{n}_{\alpha}-\gamma(\hat{\mathbf{m}} \cdot \mathbf{n}) v_{\alpha}^{\mathrm{slip}}=\lambda^{\prime} n_{\alpha},
$$

where $\lambda^{\prime}$ is a Lagrange multiplier due to the normalization condition of the surface director.

We would like to point out that the boundary conditions derived above can be obtained as well through an equivalent variational approach using a functional composed of the dissipation functional and the rate of change of the free energy, following the Onsager principle as applied in Ref. [11]. It can also yield the bulk hydrodynamic equations simultaneously. In our derivation, similar to the variational derivation of the Navier boundary condition for simple fluids, both the surface director and velocity field are first diluted in a thin boundary layer and then attributed to an ideal plane. Such an assumption is necessary for dealing with surface effects within a coherent continuum theory. It is analyzed in Ref. [4] for a nematic boundary layer and proven to rest on firm ground even when the boundary layer thickness is comparable to the molecular length scale.

\section{NUMERICAL SIMULATION OF BISTABLE TWISTED NEMATIC CELL}

With the setup of our boundary conditions appropriate for realistic nematic cells, we are able to explore the thus far unexplored areas of NLC hydrodynamics, such as the effects of slippage, weak anchoring, and the dissipative translationrotation coupling at the surface. To be specific, we consider a nematic cell sandwiched between two plates perpendicular to the $z$-axis, with the upper plate located at $z=d / 2$ and the lower plate at $z=-d / 2$. In this first approach, we investigate numerically the one-dimensional (1D) hydrodynamics in which the NLC layer is assumed to be homogeneous in the $x$ and $y$ directions and all the involved quantities vary only along the $z$ axis. A 2D model is under development which will enable us to explore the effects of textured substrate. Note that in our model both the director and velocity are three-component vectors: $\mathbf{n}=\left\{n_{x}, n_{y}, n_{z}\right\}$ and $\mathbf{v}=\left\{v_{x}, v_{y}, v_{z}\right\}$.

Following the generalized weak anchoring potential proposed recently $[13,14]$, we model the anchoring potential by $W^{ \pm}=\left[W_{1}^{ \pm}\left(\mathbf{n} \cdot \mathbf{n}_{1}^{ \pm}\right)^{2}+W_{2}^{ \pm}\left(\mathbf{n} \cdot \mathbf{n}_{2}^{ \pm}\right)^{2}\right] / 2$, with $\mathbf{n}_{1}^{ \pm}=\left(\mathbf{e}^{ \pm} \times \hat{\mathbf{z}}\right) / / \mathbf{e}^{ \pm}$ $\times \hat{\mathbf{z}} \mid$ and $\mathbf{n}_{2}^{ \pm}=\mathbf{e}^{ \pm} \times \mathbf{n}_{1}^{ \pm}$, where $\mathbf{e}^{ \pm}$is the monostable easy axis for the corresponding substrate, with "+" for the upper surface and "-" for the lower surface. $W_{1}^{ \pm}$and $W_{2}^{ \pm}$are both positive and describe the azimuthal and polar anchoring strengths, respectively.

In order to describe the effects due to the electric field, we follow the formalism in Ref. [15], where the field contribution to the free energy density is written as

$$
F_{e f}=\frac{1}{2} \frac{D_{z}^{2}}{\epsilon_{\perp}\left(1+\frac{\epsilon_{\|}-\epsilon_{\perp}}{\epsilon_{\perp}} n_{z}^{2}\right)},
$$

in which $\epsilon_{\|}$and $\epsilon_{\perp}$ are the principal components of the lowfrequency permittivity tensor. The voltage drop $V$ across the NLC layer is related to the $z$ component of electric displacement $D_{z}$ by

$$
V=\int_{-d / 2}^{d / 2} E d z=\frac{D_{z}}{\epsilon_{\perp}} \int_{-d / 2}^{d / 2}\left(1+\frac{\epsilon_{\|}-\epsilon_{\perp}}{\epsilon_{\perp}} n_{z}^{2}\right)^{-1} d z .
$$

To correctly track the dynamic effects of a time-dependent voltage pulse, we solve the director profile and the value of $D_{z}$ self-consistently at each time step. Since the Reynolds number for a NLC is usually very small $\left(\sim 10^{-4}\right)$, we can safely neglect the inertial term in the momentum equation. This means that the velocity field responds to the direct field instantaneously.

Now we employ our boundary conditions to study the hydrodynamics in a truly bistable NLC cell. Traditionally, bistable twisted nematic (BTN) liquid crystal displays operate between bistable (metastable) states with twist angles $\phi$ and $\phi+2 \pi$, which have distinct optical transmission properties $[16,17]$. However, associated with the two operating modes, the intermediate twist state of $\phi+\pi$ usually has the lowest energy and the relaxation of the system toward this state is unavoidable for sufficiently long time [15]. Recently, Dozov et al. has demonstrated [6] electric-field-driven switching between topologically nonequivalent $\phi$ and $\phi+\pi$ twist states by posing strong and weak surface anchoring at two plates, respectively. A geometric view of the bistable configurations, the uniform state $\mathrm{U}$ and the $\pi$-twist state $\mathrm{T}$, is shown in Fig. 1. This type of BTN $(\pi$-BTN $)$ is much more stable because it switches between two configurations with the lowest free energies. The switching between the two topologically nonequivalent states is possible only by breaking surface anchoring $[7,9,10]$. As the surface effect is predominant, a satisfactory quantitative simulation of the switching process is only possible with our newly derived boundary conditions. Below we present the results of numerical simulation for the NLC dynamics during the bidirectional $\phi$ to $\phi+\pi$ switching. The simulation was implemented with the time-domain finite-difference method [16,17].

First we specify the viscoelastic, dielectric, geometric, and NLC-solid interfacial parameters for our system. The bulk viscosity coefficients and elastic constants are taken from Ref. [17], and are appropriate for methoxybenzilidene butylanaline (MBBA). We use the dielectric constants $\epsilon_{\|}$ $=19$ and $\epsilon_{\perp}=6$. The cell thickness $d$ is set to be $1.5 \mu \mathrm{m}$. The actual value of the twist angle $\phi$ is found to be of little influence on the dynamic responses of the system. We thus choose $\phi=0$. Correspondingly, we set $\mathbf{e}^{-}=(\cos \theta, 0, \sin \theta)$ and $\mathbf{e}^{+}=\left(\cos 5^{\circ}, 0, \sin 5^{\circ}\right)$, where the pretilt angle for the upper plate is fixed at $5^{\circ}$ and that for the lower plate $\theta$ should be extremely small (below $1^{\circ}$ ) for reasons to be clarified later. The anchoring coefficients are chosen to be reasonably large so as to be easily realized in experiments [18]: $W_{1}$ $=0.033, W_{2}^{-}=0.2, W_{1}^{+}=0.23$, and $W_{2}^{+}=1.5$, in units of erg $/ \mathrm{cm}^{2}$ 
(a)

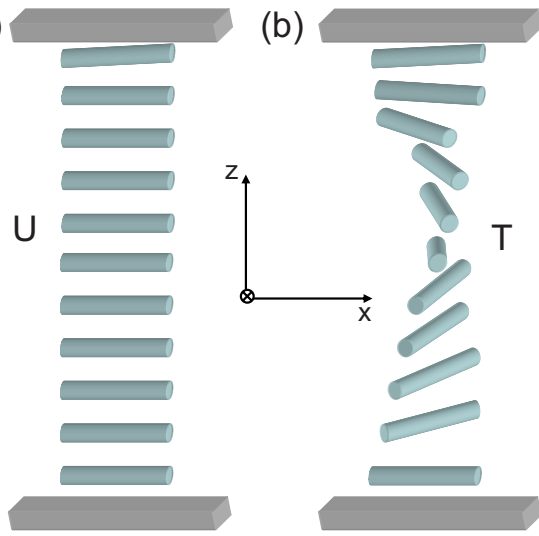

FIG. 1. (Color online) Schematic view of the bistable $\pi$-twist nematic liquid crystal cell. The NLC sample is sandwiched by two plates, with stronger anchoring at the upper plate and weaker anchoring at the lower plate. It switches between two topologically nonequivalent configurations with the lowest energies: (a) the uniform state $\mathrm{U}$ and (b) the $\pi$-twist state $\mathrm{T}$. Switching from $\mathrm{U}$ to $\mathrm{T}$ is realized by applying an electric field large enough to break the anchoring at the lower plate and switching off the field suddenly to get the surface director flipped via the backflow effect. The reverse switching requires a slow switching off of the electric field to prevent the backflow effect. A two-step voltage pulse is often used for this purpose.

or $\mathrm{mJ} / \mathrm{m}^{2}$. The polar anchoring strength for the relatively weak anchoring at the lower plate $\left(W_{2}^{-}\right)$corresponds to an extrapolation length $L_{W}$ of about $50 \mathrm{~nm}$ (or $1 / 30$ of the cell thickness). The doping pitch $p$ should also be carefully chosen so that the free energies of $\phi$ and $\phi+\pi$ states are the lowest and close to each other. From a heuristic point of view, the ideal $d / p$ ratio is 0.25 for the zero- and $\pi$-twist states to have approximately the same elastic free energy.
However, a larger $d / p$ ratio is often used to balance the elastic energies of the two twist states. Here $d / p=0.33$ is used. In fact, our study shows that the critical electric field required to realize the $\pi$-BTN depends little on the $d / p$ ratio as the switching process is mostly determined by the surface dynamic behavior.

Surface parameters are to be provided for implementing the general boundary conditions. Due to the lack of experimental data for the slip length $l_{s}$ and rotational friction length $l_{r}$ at the NLC-solid interface, we set $l_{s}=l_{r}=15 \mathrm{~nm}$, which are considered to be in a reasonable range [19]. The ratio of these two lengths is more important, as it provides an upper limit for the dissipative translation-rotation coupling coefficient $\gamma$. For example, with the bulk viscosities for MBBA, $l_{s}=l_{r}$ requires $\gamma<0.75 \gamma_{1}$ (with $\alpha_{4} \simeq 1.1 \gamma_{1}$ ), while $l_{r}=100 l_{s}$ requires $\gamma<7.5 \gamma_{1}$. The inequality given by Eq. (8) proves to be very important to ensure the existence of stable solutions.

For convenience and clearness, we use effective units to dedimensionalize our equations. We choose $d=1.5 \mu \mathrm{m}$ as the length unit, $K^{*}=10^{-6} \mathrm{erg} / \mathrm{cm}$ the unit for elastic constants, and $\gamma_{1}$ the unit for all the bulk viscosities and the surface frictional parameter $\gamma$. The time unit is $\gamma_{1} d^{2} / K^{*}$ $\simeq 9.5 \mathrm{~ms}$, while the velocity unit is approximately $0.158 \mathrm{~mm} / \mathrm{s}$. Effective units are assumed afterward if not specified, while voltages are always measured in volts. In a recent model [4] for surface director relaxation, where there is no slip and no dissipative translation-rotation coupling $(\gamma=0), l_{r}$ is shown to be quite independent of the extrapolation length $L_{W}$, and the surface relaxation time is given by $\tau_{s} \simeq \gamma_{1} L_{W} l_{r} / K^{*}$. With $l_{r}$ nearly six times smaller and $\gamma_{1}$ three times larger compared to the corresponding parameters used in Ref. [7], and taking into account that the value of $K^{*}$ overestimates the average elastic constant by several times, we roughly obtain $\tau_{s} \sim 10^{-5} \mathrm{~s}$ for the lower surface, at the same order as the value given by Ref. [7]. With nonzero
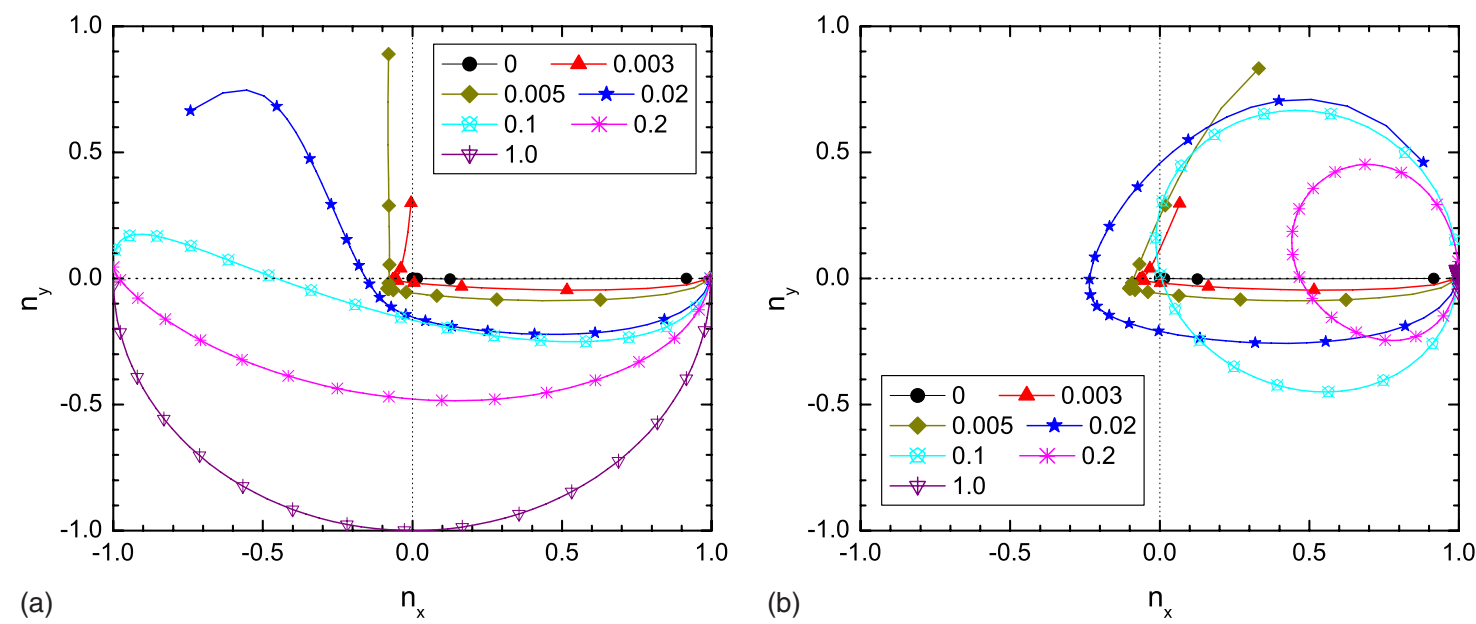

FIG. 2. (Color online) A voltage pulse $\left(V_{1}, \tau_{1}\right)$ is applied to the nematic cell initially in the $\mathrm{U}$ state. Here we show the evolution of the director configuration starting from the end of the pulse $(t=0)$. We choose 17 equally spaced points from the lower plate $(z=-d / 2)$ to the upper plate $(z=d / 2)$, project the director at each point to the $\left(n_{x}, n_{y}\right)$ plane, and connect these points according to the spatial order to represent the director configuration in the whole cell. In the $\mathrm{U}$ state, the $n_{x}$ component near the upper plate $(z=d / 2)$ is always close to 1 since the applied voltage is not large enough to break the anchoring there. The pretilt angle at the lower plate is $\theta=0.5^{\circ}$ and the cross-coupling constant is $\gamma=0.5$. In this and all the other figures, $\gamma$ is always measured in units of $\gamma_{1}$. (a) For $\left(V_{1}, \tau_{1}\right)=(27.8,0.01)$, the applied pulse switches the initial $\mathrm{U}$ state to the $\mathrm{T}$ state. (b) For $\left(V_{1}, \tau_{1}\right)=(27.7,0.01)$, the applied voltage is not strong enough to induce a switching from $\mathrm{U}$ to $\mathrm{T}$ : the system relaxes back to the $\mathrm{U}$ state via an intermediate $2 \pi$-twist state. 


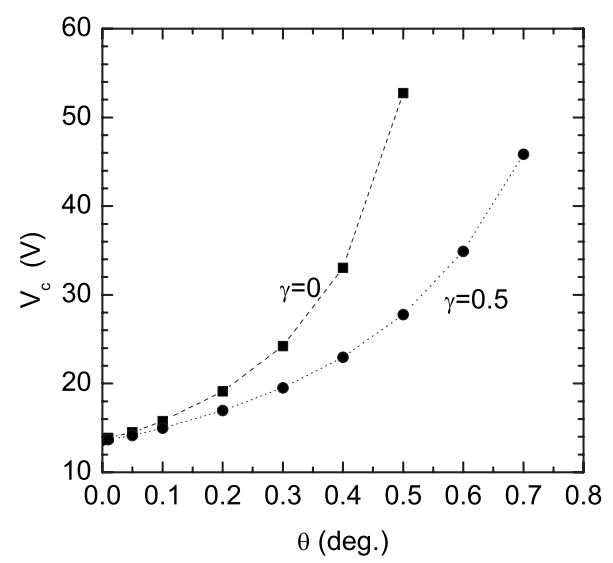

FIG. 3. Critical voltage $V_{c}$ for the switching from $\mathrm{U}$ to $\mathrm{T}$ is plotted as a function of the pretilt angle $\theta$ at the lower surface, for $\gamma=0$ (dashed line) and 0.5 (dotted line). Here $\theta$ is kept smaller than $1^{\circ}$ so that $V_{c}$ is not too large.

dissipative translation-rotation coupling, the surface director's relaxation becomes even faster and can be faster than the bulk relaxation by four orders of magnitude.

To switch from the zero-twist (or U for "uniform") to the $\pi$-twist (or T for "twist") state, a voltage pulse of rectangular wave form is applied for a time duration $\tau_{1}$ and then turned off. The applied pulse amplitude $V_{1}$ should exceed a critical voltage $V_{c}$ in order to break the anchoring at the lower surface and induce a backflow that is large enough to flip the director there. Figure 2(a) shows the simulated evolution starting from an initial $\mathrm{U}$ state immediately after a voltage pulse $\left(V_{1}, \tau_{1}\right)=(27.8,0.01)$ is applied, while Fig. 2(b) shows the evolution from the same state after a lower voltage 27.7, slightly below $V_{c}=27.8$, is applied for the same time duration $\tau_{1}$. While both cases show the anchoring at the lower surface to be broken, the dynamic processes and the corresponding final states are very different. At a rather early moment $(t$ $\sim 0.003$ ), qualitative differences can already be clearly seen between the surface director configurations in Figs. 2(a) and

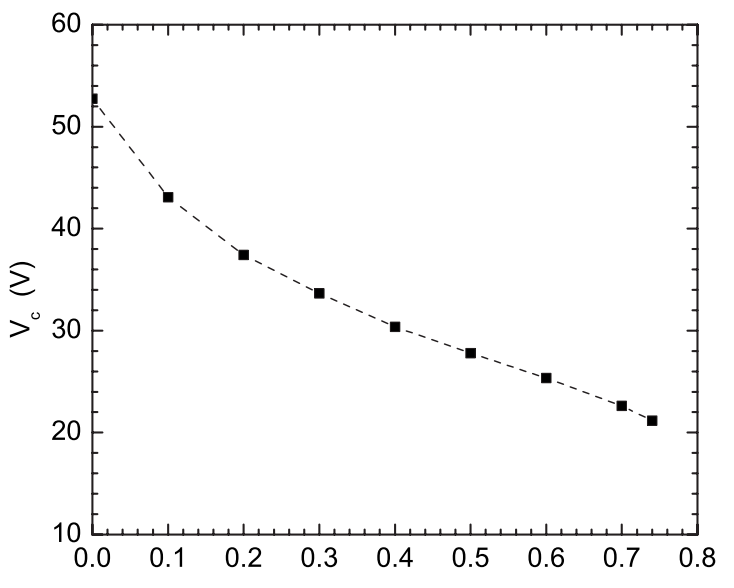

(a)

$\gamma$ 2(b). Far away from the lower surface, there is no obvious difference. In Fig. 2(a), the backflow near the lower surface is strong enough to flip the sign of the $x$ component of the surface director, and this component keeps decreasing afterward, i.e., it relaxes in the opposite direction with respect to the upper part of the cell. As a result, the whole cell develops into a $\pi$-bend state. After a sufficiently long time $(t \sim 0.2)$, the $\pi$-bend state relaxes into the desired $\mathrm{T}$ state with a lower elastic energy. On the contrary, in Fig. 2(b) the surface backflow is not so strong and the $x$ component of the surface director never gets flipped even though it can get very close to zero. Due to the backflow in the middle part of the cell, it first evolves into a nearly $2 \pi$-twist state and then relaxes back toward the initial zero-twist state with a lower energy.

We find that the critical voltage $V_{c}$ for the switching from $\mathrm{U}$ to $\mathrm{T}$ is highly sensitive to the pretilt angle $\theta$ at the lower surface. In Fig. 3 we plot $V_{c}$ as a function of $\theta$, for $\gamma=0$ and $\gamma=0.5$, respectively. First we note that, given the same $\theta, \gamma$ $=0.5$ always leads to a lower $V_{c}$ than $\gamma=0$. In particular, this difference in $V_{c}$ increases with the increasing $\theta$. At $\theta=0.5^{\circ}$, the critical voltage for $\gamma=0.5$ is nearly half of that for $\gamma=0$. In both cases, a slight increase of $\theta$ leads to an exponential increase of $V_{c}$. By analyzing the very early stage of the dynamic process, we find that a change of $\theta$ from $0.3^{\circ}$ to $0.31^{\circ}$ can cause a nearly $10 \%$ reduction in the rate of surface relaxation, leading to very different scenarios eventually, even though at the early stage the director and flow fields for the two different pretilt angles show little difference.

In Fig. 4(a) we plot $V_{c}$ for the switching from $\mathrm{U}$ to $\mathrm{T}$ as a function of $\gamma$, with all the other parameters remaining the same as in Fig. 2(a). It is seen that $V_{c}$ decreases monotonically with the increasing cross coupling constant $\gamma$. This is physically reasonable considering that $\gamma$ is a parameter describing the coupling between the translational and rotational fluxes at the surface, and this coupling would facilitate the cooperation between director rotation and mass transport to reduce the energy dissipation according to the Onsager variational principle of least energy dissipation [5]. To quantitatively investigate the role of $\gamma$, we plot in Fig. 4(b) the $x$

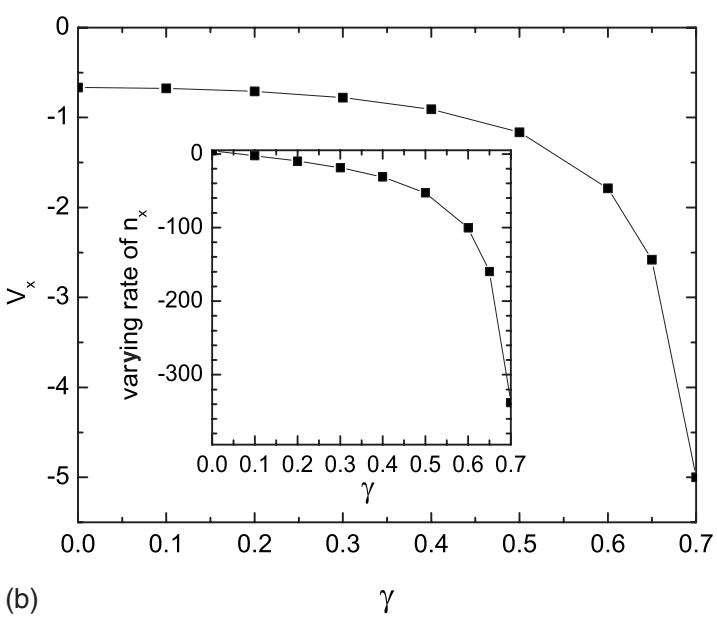

FIG. 4. (a) Critical voltage $V_{c}$ is plotted as a function of $\gamma$ for $\theta=0.5^{\circ}$. Note that Eq. (8) gives 0.75 as the upper bound of $\gamma$ for the parameters we have chosen. (b) The $x$ component of fluid velocity at the lower surface is plotted as a function of $\gamma$, immediately after the pulse $\left(V_{1}, \tau_{1}\right)=(27.8,0.01)$ is applied to possibly induce the switching from $\mathrm{U}$ to $\mathrm{T}$. The inset shows the corresponding relaxation rate of $n_{x}$, i.e., $\dot{n}_{x}$ at the lower surface at the same moment, as a function of $\gamma$. 
component of fluid velocity $V_{x}$ and the corresponding rate of director relaxation $\dot{n}_{x}$ at the lower surface immediately after the applied voltage pulse $\left(V_{1}, \tau_{1}\right)$ is turned off. It is shown that $V_{x}$ is negative and its magnitude increases monotonously with the increasing $\gamma$. It is also observed (not shown here) that $V_{y}$ (about two orders of smaller than $\left|V_{x}\right|$ ) is positive and also increases monotonically with increasing $\gamma$. Thus a larger $\gamma$ tends to enhance the backflow effect and hence lower the critical voltage. From the inset of Fig. 4(b) we can see that as $\gamma$ is increased, $\dot{n}_{x}$ becomes more negative and the flipping of $n_{x}$ is thus accelerated. It is noted that as $\gamma$ approaches its upper bound ( 0.75 here) set by the inequality in Eq. (8), both the flow velocity and director relaxation rate would blow up exponentially.

To realize the switching from $\mathrm{T}$ to $\mathrm{U}$, a relatively lower voltage (but still higher than the static threshold to break the anchoring at the lower plate) can be used to erase the memory of the $\mathrm{T}$ state. The voltage cannot be switched off abruptly, since the backflow effect should be restrained. A two-step pulse of rectangular wave form can be used to realize the $\mathrm{T}$ to $\mathrm{U}$ switching, with the first pulse $\left(V_{1}^{\prime}, \tau_{1}^{\prime}\right)$ immediately followed by the second pulse $\left(V_{2}, \tau_{2}\right)$ weaker than the first. We plot in Fig. 5 the director histogram of the NLC cell starting from the end of the second pulse $(t=0.001)$. It is interesting to note that, similar to the evolution of a $U$ state subject to a voltage below $V_{c}$ [cf. Fig. 2(b)], the memoryerased $\mathrm{T}$ state (at $t=0$ ) first relaxes into a $2 \pi$-twist state, which eventually develops into the final zero-twist $U$ state with a lower elastic energy. The duration of $\tau_{2}$ could be as small as 0.0001 , or approximately $1 \mu \mathrm{s}$, which is much less than the estimated value, $50 \sim 100 \mu$ s, given in Ref. [7]. This is understandable since the surface relaxation in our system is much faster. A nonzero translation-rotation coupling $\gamma$ $>0$ has a twofold effect on the switching process from the $\mathrm{T}$ to the U state: on one hand, it enhances the backflow and tends to suppress the transition; on the other hand, it facilitates the surface relaxation and may allow a faster switching.

\section{CONCLUSIONS}

In conclusion, we have developed a set of general boundary conditions to describe the NLC dynamics at solid surface, taking into account a weak anchoring, slippage and the dissipative cross coupling between the translational and rotational degrees of freedom. We have applied our boundary

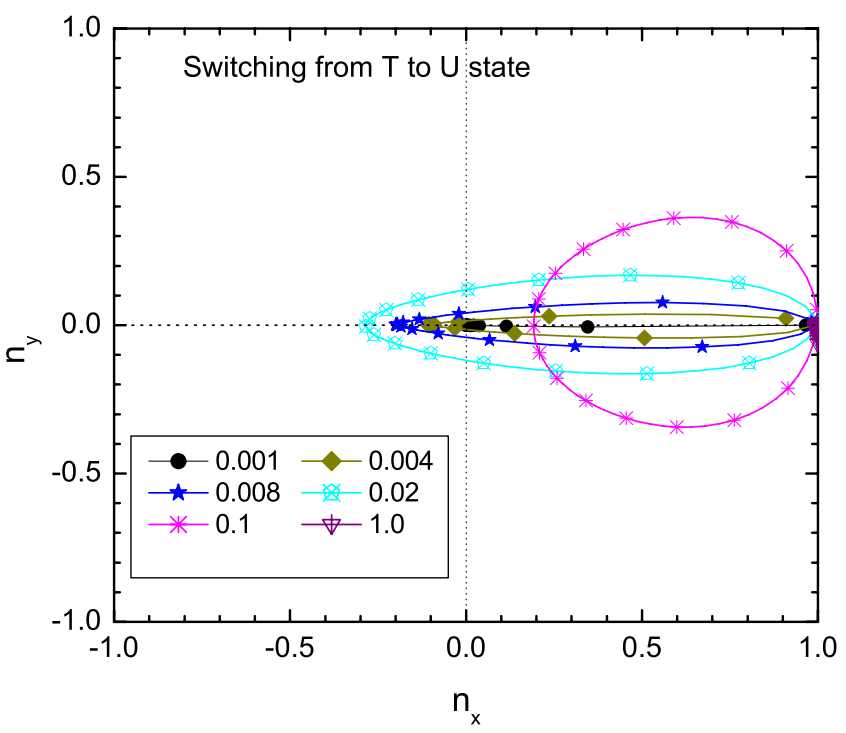

FIG. 5. (Color online) A two-step voltage pulse $(27.8,0.01)$ followed immediately by $(13.9,0.001)$ is applied to the nematic cell initially $(t=-0.01)$ in the T state. Here we show the evolution of the director configuration starting from the end of the second rectangular wave form $(t=0.001)$. All the parameters are the same as for Fig. 2 . The system relaxes to the zero-twist $U$ state via an intermediate $2 \pi$-twist state.

conditions to the switching process in a $\pi$-BTN cell, in which anchoring breaking is essential and surface dynamics plays a dominant role. We find that the translation-rotation coupling at the surface can significantly influence the switching dynamics and properly chosen material parameters can greatly improve the performance of electric-optical devices by lowering the critical switching voltage and accelerating the switching process. It is of interest to apply the general boundary conditions to a vast category of hydrodynamical phenomena of liquid crystals where surface effects are important, e.g., the electric-field controlled switching of multistable NLC induced by textured substrate [20].

\section{ACKNOWLEDGMENTS}

This work was supported by Hong Kong RGC Grant No. CA05/06.SC01. A.F. acknowledges support from the KAUST Global Research Partnership. T.Q. was also supported by Hong Kong RGC Grant No. 602007.
[1] P. G. de Gennes and J. Prost, The Physics of Liquid Crystals (Oxford University Press, New York, 1993).

[2] A. I. Derzhanski and A. G. Petrov, Acta Phys. Pol. A 55, 747 (1979).

[3] M. Kléman and S. A. Pikin, J. Mec. 18, 661 (1979).

[4] A. M. Sonnet and E. G. Virga, Phys. Rev. E 61, 5401 (2000); A. M. Sonnet, E. G. Virga, and G. E. Durand, ibid. 62, 3694 (2000).

[5] L. Onsager, Phys. Rev. 37, 405 (1931); 38, 2265 (1931); L.
Onsager and S. Machlup, ibid. 91, 1505 (1953).

[6] I. Dozov, M. Nobili, and G. Durand, Appl. Phys. Lett. 70, 1179 (1997).

[7] M. Giocondo, I. Lelidis, I. Dozov, and G. Durand, Eur. Phys. J.: Appl. Phys. 5, 227 (1999).

[8] J. G. McIntosh and F. M. Leslie, J. Eng. Math. 37, 129 (2000).

[9] T. Z. Qian, Z. Xie, H. S. Kwok, and P. Sheng, J. Appl. Phys. 90, 3121 (2001).

[10] T. Takemi, S. Oka, M. Kimura, and T. Akahane, Jpn. J. Appl. 
Phys., Part 1 43, 7629 (2004).

[11] T. Qian, X.-P. Wang, and P. Sheng, J. Fluid Mech. 564, 333 (2006); T. Qian, C. Qiu, and P. Sheng, ibid. 611, 333 (2008).

[12] T. Qian, X.-P. Wang, and P. Sheng, Phys. Rev. E 68, 016306 (2003); Phys. Rev. Lett. 93, 094501 (2004).

[13] T. Beica, S. Frunza, R. Moldovan, and D. N. Stoenescu, Mol. Cryst. Liq. Cryst. Sci. Technol., Sect. A 301, 39 (1997).

[14] W. Zhao, C.-X. Wu, and M. Iwamoto, Phys. Rev. E 62, R1481 (2000); 65, 031709 (2002).

[15] S. P. Palto and M. I. Barnik, JETP 100, 199 (2005).
[16] D. W. Berreman and W. R. Heffner, J. Appl. Phys. 52, 3032 (1981).

[17] T. Z. Qian, Z. L. Xie, H. S. Kwok, and P. Sheng, Appl. Phys. Lett. 71, 632 (1997).

[18] F. S. Y. Yeung and H. S. Kwok, Appl. Phys. Lett. 83, 4291 (2003).

[19] L. Blinov, G. Durand, and S. Yablonsky, J. Phys. II 2, 1287 (1992).

[20] J.-H. Kim, M. Yoneya1, and H. Yokoyama, Nature (London) 420, 159 (2002). 